\title{
A!
}

This is an electronic reprint of the original article.

This reprint may differ from the original in pagination and typographic detail.

Rimpiläinen, Tommi; Wallen, H.; Sihvola, A.

Polarizability of radially anisotropic elliptic inclusion

Published in:

Journal of Applied Physics

DOI:

$10.1063 / 1.4939158$

Published: 07/01/2016

Document Version

Publisher's PDF, also known as Version of record

Please cite the original version:

Rimpiläinen, T., Wallen, H., \& Sihvola, A. (2016). Polarizability of radially anisotropic elliptic inclusion. Journal of Applied Physics, 119(1), [014107]. https://doi.org/10.1063/1.4939158

This material is protected by copyright and other intellectual property rights, and duplication or sale of all or part of any of the repository collections is not permitted, except that material may be duplicated by you for your research use or educational purposes in electronic or print form. You must obtain permission for any other use. Electronic or print copies may not be offered, whether for sale or otherwise to anyone who is not an authorised user. 


\section{Polarizability of radially anisotropic elliptic inclusion}

T. Rimpiläinen, H. Wallén, and A. Sihvola

Citation: Journal of Applied Physics 119, 014107 (2016); doi: 10.1063/1.4939158

View online: https://doi.org/10.1063/1.4939158

View Table of Contents: http://aip.scitation.org/toc/jap/119/1

Published by the American Institute of Physics

\section{Articles you may be interested in}

Cloaking and magnifying using radial anisotropy

Journal of Applied Physics 114, 044110 (2013); 10.1063/1.4816797

Scattering of Electromagnetic Waves from Two Concentric Spheres

Journal of Applied Physics 22, 1242 (1951); 10.1063/1.1699834 


\title{
Polarizability of radially anisotropic elliptic inclusion
}

\author{
T. Rimpiläinen, ${ }^{\text {a) }} \mathrm{H}$. Wallén, and A. Sihvola \\ Department of Radio Science and Engineering, Aalto University School of Electrical Engineering, \\ P.O. Box 13000, FI-00076 Aalto, Finland
}

(Received 1 October 2015; accepted 16 December 2015; published online 7 January 2016)

\begin{abstract}
This article discusses a two-dimensional electrostatic scattering problem where an elliptic inclusion is suspended in a homogeneous background and impinged by an electric field which is uniform and static. The novelty of the discussion stems from the inclusion's material parameters. The material of the inclusion is assumed to be axially anisotropic, so that the axis of anisotropy aligns itself with the radial unit vector of the elliptic coordinate system. Similar varieties of anisotropy have been formerly referred to as radial anisotropy, and the same term is employed herein. The radially anisotropic elliptic inclusions are studied with an analytic method. The validation is likewise analytic. The validation method compares the new results with the results for radially anisotropic circles and homogeneous two-dimensional needles. The elliptic inclusion is found to facilitate both cloaking and field concentration. (C) 2016 AIP Publishing LLC. [http://dx.doi.org/10.1063/1.4939158]
\end{abstract}

\section{INTRODUCTION}

A central topic in electrostatics concerns dielectric inclusions that extend linearly to two opposite directions. If this type of inclusion is extended so far, that in an approximation it can be considered infinite, the analysis of the electrostatic scattering problem reduces into two dimensions, whereas the third can be omitted because of symmetry. The simplest of such inclusions is the infinite wire, corresponding to a point in two dimensions. Adding width while retaining the rotational symmetry gives us the infinite circular cylinder. And breaking the rotational symmetry by means of a linear elongation or compression in the cross-sectional plane leaves us with the infinite elliptic cylinder, the topic of the present paper.

There are several reasons to take interest in elliptic geometry, not the least of which relates to its applicability in the theory and technique of guided waves. ${ }^{1}$ But even when we exclude the time-variant fields from our analysis, the theory still finds its uses. Manifestations of straight, elongated shapes include engineering artifacts, like antennas and transmission lines, but also many objects of organic provenance, like trees and root vegetables. Examples can be equally found from inorganic nature, where elongated ice crystals play a contingent role in many fields of atmospheric physics.

Fortunately, ellipse's broad range of applications does not preclude mathematical tractability. Moon and Spencer ${ }^{2}$ list eleven coordinate systems that permit the Laplace equation and the Helmholtz equation to separate to ordinary differential equations. Among this restricted group, we find the system that is most pertinent for our present study, the system of elliptic-cylinder coordinates. The separability of Laplace equation in elliptic-cylinder coordinates lends this system a pivotal role in our pursuit for analytic solution for the electrostatic scattering problem. The advantages of the chosen system of coordinates become manifest when we express the electric potentials exterior and interior to the

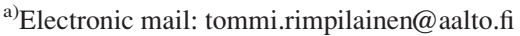

inclusion in elliptic-harmonic functions. The representation of these harmonic functions aligns with the elliptic geometry of the inclusion, whereby we can easily postulate the usual interface conditions for the electric field.

The tractable mathematical character of ellipse problems has attracted plenty of theoretical research. Former treatment of elliptic-cylinder coordinates in electromagnetic theory involve a diversity of topics, including the aforementioned guided waves, but also encompassing electrostatic Green's functions ${ }^{3,4}$ and layered non-confocal structures. ${ }^{5}$ Part of the motivation for expanding the scope of closed-form solutions is to establish a network of cross-checks, against which we can evaluate the accuracy of other methods, such as the moment method. ${ }^{6}$

The present study concerns infinite elliptic cylinders in a static excitation field. We introduce a novel variety of radial anisotropy (RA), where the axis of anisotropy is aligned with the elliptic-cylinder coordinates. We can imagine radially anisotropic ellipses as consisting of a dense set of layers with alternating material parameters. These layers align themselves with the inclusion's geometry so that each layer exactly coincides with a coordinate curve in the ellipse coordinate system and thus takes on an elliptic shape. The outermost layer exactly coincides with the elliptic surface of the inclusion. Because we assumed the stack of layers to be dense, at each point within the inclusion, the layers can be regarded planar up to a first order approximation. When we homogenize the stack of layers by averaging the pertinent fields suitably, an uniaxial anisotropy emerges. ${ }^{7}$ The asymmetry imposed by the layered structure makes the effective material parameter contingent on the orientation of the incoming electric field that the material acts upon. In quantitative terms, we express the relationship in terms of the dyadic

$$
\overline{\bar{\epsilon}}=\epsilon_{\mathrm{n}} \mathbf{u}_{\eta} \mathbf{u}_{\eta}+\epsilon_{\mathrm{t}} \mathbf{u}_{\psi} \mathbf{u}_{\psi},
$$

where $\mathbf{u}_{\eta}$ and $\mathbf{u}_{\psi}$ are the unit vectors of the elliptic coordinate system (see Fig. 1). 


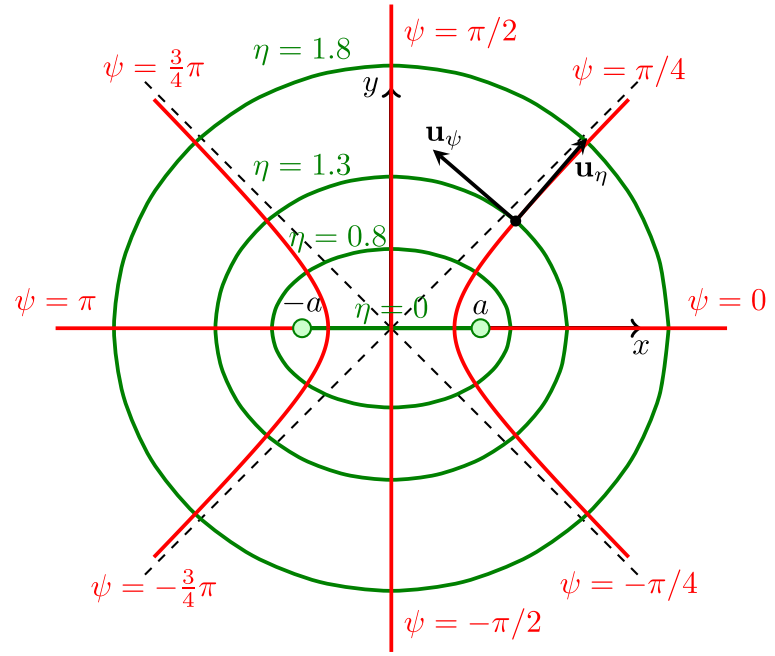

FIG. 1. System of elliptic coordinates. The coordinate curves $\eta=$ const form ellipses and the coordinate curves $\psi=$ const form hyperbolae with focal points $x=-a$ and $x=a$ in each case.

While the homogeneous elliptic cylinder has been studied for both dynamic and static fields ${ }^{5}$ and while the concept of radial anisotropy has been considered for circular cylinders 8,9 and spheres ${ }^{10-15}$ before, even in a generalized sense, ${ }^{16,17}$ the present study examines electrostatic scattering with a combination of material and geometric properties unprecedented, as far as the authors know, in the electromagnetic literature.

\section{PRELIMINARIES}

The present study concerns an elliptic inclusion suspended in a homogeneous background medium and impinged by a static, uniform excitation field $\mathbf{E}^{\mathrm{p}}$. It can be assumed, without loss of generality, that the medium outside the inclusion is vacuum because the permittivities only enter the equations in relative fractions. The dipole moment $\mathbf{p}$ of the scatterer depends on the excitation field $\mathbf{E}^{\mathrm{p}}$ linearly, whereby it can be expressed as

$$
\mathbf{p}=S \epsilon_{0} \overline{\bar{\alpha}} \cdot \mathbf{E}^{\mathrm{p}},
$$

where $S$ is the area of the inclusion and $\epsilon_{0}$ is the vacuum permittivity. The dyadic constant of proportionality $\overline{\bar{\alpha}}$ is the twodimensional normalized polarizability. In the far-region of the scatterer, the normalized polarizability stands out as the most significant contributor to the perturbation field $\mathbf{E}^{\mathrm{s}}$. The polarizability dyadic can be broken down into two components

$$
\overline{\bar{\alpha}}=\alpha_{\|} \mathbf{u}_{x} \mathbf{u}_{x}+\alpha_{\perp} \mathbf{u}_{y} \mathbf{u}_{y}
$$

so that the $\alpha_{\|}$component expresses the polarizability along the longer semi-axes of the inclusion and $\alpha_{\perp}$ along the shorter one. Here, we have tacitly aligned the Cartesian unit vectors $\mathbf{u}_{x}$ and $\mathbf{u}_{y}$ with the corresponding semi-axis of the inclusion.

\section{POLARIZABILITY OF RA ELLIPSE}

The introduction postulated ellipses as one of the electromagnetic theory's canonical shapes that find their expression in a separable coordinate system. Sec. III A discusses the suitable system of coordinates. Secs. III B and III C present a closed form solution to the electrostatic scattering problem.

\section{A. Elliptic coordinates}

The elliptic system of coordinates in Fig. 1 relates to the Cartesian system in accordance with the equation pair

$$
\begin{aligned}
& x=a \cosh \eta \cos \psi, \\
& y=a \sinh \eta \sin \psi,
\end{aligned}
$$

where the coordinate value $\eta$ varies between zero and positive infinity and the coordinate value $\psi$ between $-\pi$ and $\pi$. The choice of the coordinate $\eta=$ const determines a coordinate curve in the two dimensional plane, as does the choice of the coordinate $\psi=$ const. When different values of $\eta$ are chosen, the coordinate curves in Fig. 1 take the shape of ellipses, the axes of which expand with the increasing $\eta$ values. These ellipses are confocal so that the parameter $a$ determines the focal points $(-a, 0)$ and $(a, 0)$ in the $x y$-plane. The other set of coordinate curves, related to the different values of $\psi$, assume the shape of hyperbolae with the same focal points. With large values of $\eta$, the confocal ellipses start to resemble circles and also the coordinates of the elliptic coordinate system closely coincide with those of the polar system. The connection between these two systems of coordinates, far away from the focal points, is

$$
\begin{aligned}
& \rho \approx \frac{a e^{\eta}}{2}, \\
& \varphi \approx \psi .
\end{aligned}
$$

Because we assumed that the longer semi-axis of the inclusion falls in alignment with the Cartesian $x$-axis, there necessarily exists a parameter value $a$ that matches the focal points of the coordinate system with those of the inclusion. The ellipse surface then corresponds to some value of the radial coordinate $\eta=\eta_{0}$. If $b=a \cosh \eta_{0}$ denotes the longitudinal half semi-axis and if $c=a \sinh \eta_{0}$ denotes the lateral half semi-axis, the relationship $a^{2}=b^{2}-c^{2}$ follows from the properties of the hyperbolic trigonometric functions. The lengths $b$ and $c$ also determine the coordinate value of the inclusion's boundary: $\eta_{0}=\operatorname{artanh} c / b$.

The elliptic coordinate system allows the ordinary Laplace equation to separate, ${ }^{2}$ but since the inclusion consists of radially anisotropic material (1), the anisotropy and the fact that the inclusion is inhomogeneous need to be factored into the field equation. Therefore, the ordinary Laplace equation is not pertinent to the present scattering problem. We can construct the pertinent equation, when we note that the electric flux density in terms of the potential $\phi$, is

$$
\mathbf{D}=-\overline{\bar{\epsilon}} \cdot \nabla \phi=-\overline{\bar{\epsilon}} \cdot\left(\frac{\mathbf{u}_{\eta}}{h_{\eta}} \frac{\partial \phi}{\partial \eta}+\frac{\mathbf{u}_{\psi}}{h_{\psi}} \frac{\partial \phi}{\partial \psi}\right)
$$

and that the divergence of $\mathbf{D}$ must vanish in absence of sources 


$$
\nabla \cdot \mathbf{D}=\frac{1}{h_{\eta} h_{\psi}}\left[\frac{\partial}{\partial \eta}\left(D_{\eta} h_{\psi}\right)+\frac{\partial}{\partial \psi}\left(D_{\psi} h_{\eta}\right)\right]=0 .
$$

When we combine (6) and (7) and substitute, the values of the metric coefficients

$$
h_{\eta}=h_{\psi}=a \sqrt{\cosh ^{2} \eta-\cos ^{2} \psi}
$$

we find the generalization of the Laplace equation applicable for the radially anisotropic medium

$$
\epsilon_{\mathrm{n}} \frac{\partial^{2} \phi}{\partial \eta^{2}}+\epsilon_{\mathrm{t}} \frac{\partial^{2} \phi}{\partial \psi^{2}}=0
$$

\section{B. Parallel polarizability}

The first case we consider involves an elliptic inclusion in an excitation field which is oriented parallel to the longer semi-axis of the inclusion. As before, this direction aligns with the positive $x$-axis, so that the excitation field is $\mathbf{E}^{\mathrm{p}}=E^{\mathrm{p}} \mathbf{u}_{x}$, corresponding to the potential

$$
\phi^{\mathrm{p}}=-E^{\mathrm{p}} x=-U_{0} \cosh \eta \cos \psi,
$$

where we use the shorthand $U_{0}=a E^{\mathrm{p}}$. Besides this excitation field that we assume to be known, there remain two unknown potentials to be solved, the internal potential $\phi_{\text {in }}$ and the perturbation potential $\phi^{\mathrm{s}}$, as shown in Fig. 2.

A general solution to the Laplace equation (9) follows, when we substitute the Ansatz $\phi(\eta, \psi)=H(\eta) \Psi(\psi)$ and combine all the Ansatz solutions in a series. However, a simpler solution suffices for the present purposes, because of two limiting conditions. First, the angular dependence $\Psi(\psi)$ of the internal potential $\phi_{\text {in }}$ and the perturbation potential $\phi^{\mathrm{s}}$ have to correspond with the angular dependence of the excitation potential $\phi^{\mathrm{p}}$. Second, we require that the fields $-\nabla \phi_{\text {in }}$ and $-\nabla \phi^{\mathrm{s}}$ need to remain finite in their respective domains and that the last one of these vanishes infinitely far away

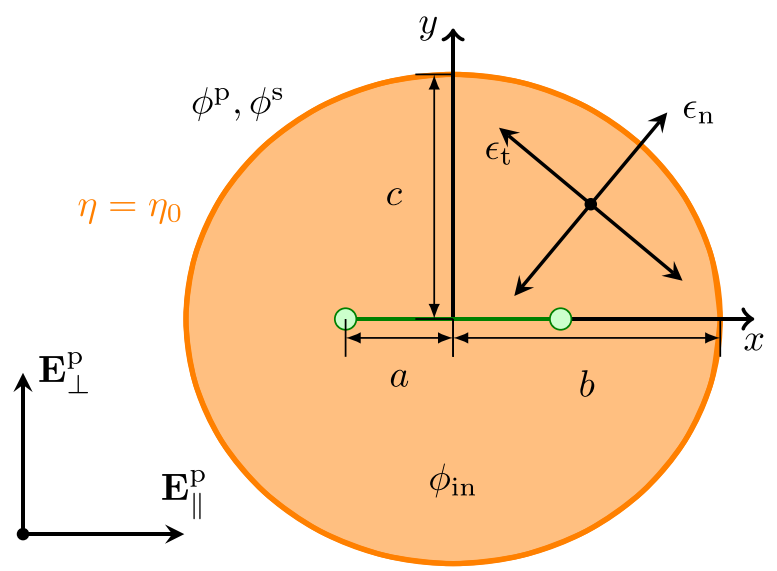

FIG. 2. Inclusion in an excitation field $\mathbf{E}^{\mathrm{p}}$. The surface of the inclusion aligns itself with the elliptic coordinate curve $\eta=\eta_{0}$. The excitation field can be decomposed into parallel $\mathbf{E}_{\|}^{\mathrm{p}}$ and perpendicular $\mathbf{E}_{\perp}^{\mathrm{p}}$ components. The excitation field gives rise to the potential $\phi_{\text {in }}$ inside the inclusion. The secondary sources created inside the inclusion give rise to the perturbation potential $\phi^{\mathrm{s}}$. The inclusion's permittivity is $\epsilon_{\mathrm{n}}$ in the direction of $\mathbf{u}_{\eta}$ and $\epsilon_{\mathrm{t}}$ in the direction of $\mathbf{u}_{\psi}$. from the inclusion. When we impose the conditions of matching angular dependency and finiteness, we are left with a solution

$$
\begin{aligned}
\phi^{\mathrm{s}} & =U_{0} A e^{-\eta} \cos \psi, \\
\phi_{\text {in }} & =U_{0} B \cosh (\mu \eta) \cos \psi,
\end{aligned}
$$

where $\mu$ is defined as $\mu=\left(\epsilon_{\mathrm{t}} / \epsilon_{\mathrm{n}}\right)^{1 / 2}$.

From (11), we see that a single constant $A$ completely determines the perturbation field. We would, however, like to express the magnitude of perturbation in terms of the normalized polarizability $\alpha_{\perp}$. For that, we observe that the perturbation potential in (11) must coincide with the potential $\phi^{\mathrm{d}}$ produced by the dipole (2) when the point of observation is far away from the inclusion

$$
\phi^{\mathrm{s}} \approx \phi^{\mathrm{d}}=\frac{p \cos \varphi}{2 \pi \epsilon_{0} \rho} .
$$

The last one of the potentials, $\phi^{\mathrm{d}}$, is expressed in the polar coordinates $(\rho, \varphi)$ where the connection to elliptic system is given by (5). When we combine (2), (5), and (12) we are left with the following connection:

$$
\alpha_{\|}=\frac{1-r^{2}}{r} A,
$$

where $r$ is the axis ratio $r=c / b=\tanh \eta_{0}$.

From (12) we see that finding the polarizability $\alpha_{\|}$ involves finding the magnitude $A$ of the perturbation field in (11). The two unknowns in (11) follow from the two usual interface conditions in electrostatics, one for the continuity of the potential $\phi^{\mathrm{p}}+\phi^{\mathrm{s}}=\phi_{\text {in }}$ and the other for the continuity of the normal component of the electric flux density $\mathbf{n} \cdot \nabla \phi^{\mathrm{p}}+\mathbf{n} \cdot \nabla \phi^{\mathrm{s}}=\epsilon_{\mathrm{n}} \mathbf{n} \cdot \nabla \phi_{\text {in }}$. When we solve the resulting pair of equations for the coefficient $A$ and use the connection (13) between $A$ and $\alpha_{\|}$, we discover the formula for normalized polarizability

$$
\alpha_{\|}=\left(1+\frac{1}{r}\right) \frac{\sqrt{\epsilon_{\mathrm{n}} \epsilon_{\mathrm{t}}} \sinh \left(\mu \eta_{0}\right)-r \cosh \left(\mu \eta_{0}\right)}{\sqrt{\epsilon_{\mathrm{n}} \epsilon_{\mathrm{t}}} \sinh \left(\mu \eta_{0}\right)+\cosh \left(\mu \eta_{0}\right)},
$$

where $\eta_{0}$ is the radial elliptic coordinate that designates the surface of the inclusion, as in Sec. III A.

\section{Perpendicular polarizability}

Turning now to the case where the excitation field is perpendicular $\mathbf{E}^{\mathrm{p}}=E^{\mathrm{p}} \mathbf{u}_{y}$ and the excitation potential is $-U_{0} \sinh \eta \sin \psi$, the new angular dependence $\sin \psi$ implies an Ansatz

$$
\begin{aligned}
\phi^{\mathrm{s}} & =U_{0} A e^{-\eta} \sin \psi, \\
\phi_{\text {in }} & =U_{0} B \sinh (\mu \eta) \sin \psi,
\end{aligned}
$$

which is slightly different than the one used in Sec. III B. The derivation of the perpendicular polarizability follows the same course as in the parallel case in Sec. III B. Because $\sinh \eta$ and $\cosh \eta$ functions take increasingly coinciding values at increasing values of $\eta$, the formerly established connection (13) between the polarizability and the coefficient $A$ 
still holds. When we impose the interface conditions at the surface of the inclusion, we find the perpendicular polarizability

$$
\alpha_{\perp}=\left(1+\frac{1}{r}\right) \frac{r \sqrt{\epsilon_{\mathrm{n}}} \epsilon_{\mathrm{t}} \cosh \left(\mu \eta_{0}\right)-\sinh \left(\mu \eta_{0}\right)}{\sqrt{\epsilon_{\mathrm{n}} \epsilon_{\mathrm{t}}} \cosh \left(\mu \eta_{0}\right)+\sinh \left(\mu \eta_{0}\right)} .
$$

\section{VALIDATION}

In this section, we validate the results by first simplifying the material parameters, in Sec. IV A. Then, we consider the simplification of geometry into a needle, in Sec. IV B, and a circle, in Sec. IV C. Numerical validation can be found in the supplementary material. ${ }^{18}$

\section{A. Isotropic ellipse}

The formula that informs the validation is the formula for the polarizability of the isotropic, homogeneous ellipsoid in three dimensions ${ }^{19}$

$$
\overline{\bar{\alpha}}_{\| / \perp}=\frac{\epsilon-1}{1+N_{x / y}(\epsilon-1)} .
$$

We apply the general formula in the case where two of the axes have a finite length and the third extends to infinity. In that case, the depolarization coefficients are

$$
N_{x}=\frac{c}{b+c}, \quad N_{y}=\frac{b}{b+c} .
$$

If we use the earlier definition $r=c / b$ and substitute the depolarization constants in the polarizability equation, we obtain the parallel polarization

$$
\alpha_{\|}=(1+r) \frac{\epsilon-1}{r \epsilon+1},
$$

and the transverse polarization

$$
\alpha_{\perp}=(1+r) \frac{\epsilon-1}{\epsilon+r} .
$$

It turns out that these equations conform exactly with (14) and (16) when we substitute the conditions for homogeneity, $\epsilon_{\mathrm{n}}=\epsilon_{\mathrm{t}}$ and ipso facto $\mu=1$, and infer from the definition of $\eta_{0}$ that $\sinh \eta_{0} / \cosh \eta_{0}=r$.

\section{B. Needle}

Another approach is to achieve the effective homogeneity by geometrical means. Let us assume that the inclusion is elongated into an $x$-directed needle. In that case, the axes of anisotropy $\mathbf{u}_{\eta}$ and $\mathbf{u}_{\psi}$ correspond to the Cartesian directions $\mathbf{u}_{y}$ and $\mathbf{u}_{x}$ nearly throughout the area of the inclusion, excepting the diminishing stretch from a focal point to the tip of the inclusion. Therefore, the parallel excitation field $\mathbf{E}_{\|}^{\mathrm{p}}$ only sees the permittivity component $\epsilon_{\mathrm{t}}$ and the transverse excitation field $\mathbf{E}_{\perp}^{\mathrm{p}}$ only sees the component $\epsilon_{\mathrm{n}}$, and consequently, we can compare the inclusion to the homogeneous ellipse, even if the inclusion is manifestly anisotropic, $\epsilon_{\mathrm{n}} \neq \epsilon_{\mathrm{t}}$.
When we let the ellipse transform into a needle, we find that the parameter $\eta_{0}$ approaches zero. In this limit, the relations $\sinh \left(\mu \eta_{0}\right) \rightarrow \mu \eta_{0}, \cosh \left(\mu \eta_{0}\right) \rightarrow 1$ and $r=\tanh \left(\eta_{0}\right) \rightarrow$ $\eta_{0}$ hold asymptotically. These asymptotic relations transform the two polarizability equations (14) and (16) into

$$
\begin{gathered}
\alpha_{\|}=\epsilon_{\mathrm{t}}-1, \\
\alpha_{\perp}=1-\epsilon_{\mathrm{n}}^{-1},
\end{gathered}
$$

which is the result anticipated by the homogeneous inclusion's polarizability equations (19) and (20).

\section{RA circle}

The second case of validation involves a radially anisotropic circle, which emerges straightforwardly as a special case of the ellipsoid when we let the lengths of the semi-axes $b$ and $c$ coincide. We can then employ the polar coordinate system and find the solution for the polarizability. ${ }^{20}$ For a circle, the polarizability is isotropic, $\overline{\bar{\alpha}}=\alpha \overline{\bar{I}}$, so that a single polarizability component $\alpha$ suffices. The polarizability of a circle is

$$
\alpha=2 \frac{\sqrt{\epsilon_{\mathrm{n}} \epsilon_{\mathrm{t}}}-1}{\sqrt{\epsilon_{\mathrm{n}} \epsilon_{\mathrm{t}}}+1} .
$$

When we let the axis ratio $r$ approach 1 , the parameter $\eta_{0}$ approaches positive infinity in the polarizability equations (14) and (16). In that limit, $\sinh \left(\mu \eta_{0}\right) \rightarrow \cosh \left(\mu \eta_{0}\right)$. By a substitution, we recover (22) in both cases as we expected.

\section{POTENTIAL IN FOCAL RANGE}

The focal range of the inclusion exhibits the curious property that, whereas the internal field of the inclusion in general varies, it remains constant between the focal points. The same property applies regardless of the orientation of the excitation field, given that the excitation remains constant and uniform, as we assumed.

To derive the field at the focal range, we consider the two internal potentials in (11) and (15) so that we take the limit $\eta \rightarrow 0$, which gives the focal range. In the first case that we consider, the excitation field is parallel. It turns out that the value of the focal electric field in the limit $\eta \rightarrow 0$ depends on the coordinate $\psi$ so that when $\psi \neq 0, \pi$, we find the limit

$$
\mathbf{E}_{\mathrm{foc}, \|}=-\nabla\left(U_{0} B \cosh (\mu \eta) \cos \psi\right) \rightarrow-B E^{\mathrm{p}} \mathbf{u}_{x},
$$

whereas the choices $\psi=0, \pi$ lead to the limit

$$
\mathbf{E}_{\text {point }, \|}=-\nabla\left(U_{0} B \cosh (\mu \eta) \cos \psi\right) \rightarrow-B \mu^{2} E^{\mathrm{p}} \mathbf{u}_{x} .
$$

In the perpendicular case, we find that the limit of $\mathbf{E}_{\mathrm{in}}(\eta, \psi)$ when $\eta \rightarrow 0$ is always

$$
\mathbf{E}_{\mathrm{foc}, \perp}=-\nabla\left(U_{0} B \sinh (\mu \eta) \sin \psi\right) \rightarrow-B \mu E^{\mathrm{p}} \mathbf{u}_{y},
$$

regardless of the coordinate value $\psi$, implying that $\mathbf{E}_{\text {foc }, \perp}=\mathbf{E}_{\text {point }, \perp}$. We will now turn to the interpretation of the results (23)-(25). 
The axis of anisotropy is fixed to the elliptic coordinate system which has two singular points at the two focal points of the ellipse that defines the inclusion. If we put our point of observation in the longitudinal axis of the inclusion but outside the focal range - that is, between $x \in(-b,-a)$ or $x \in$ $(a, b)$-we have $\mathbf{u}_{\eta}=\mp \mathbf{u}_{x}$ and $\mathbf{u}_{\psi}=\mp \mathbf{u}_{y}$. But if we put the point of observation within the focal range, $x \in(-a, a)$, we have $\mathbf{u}_{\eta}= \pm \mathbf{u}_{y}$ and $\mathbf{u}_{\psi}=\mp \mathbf{u}_{x}$.

When the field is parallel and pointed to the positive $\mathbf{u}_{x}$ direction, the only effective permittivity component has its axis aligned with $\mathbf{u}_{x}$. So, in the left to right progression along the inclusions major axis, the field first sees the permittivity component $\epsilon_{\mathrm{n}}$, then $\epsilon_{\mathrm{t}}$ in the focal range, and the $\epsilon_{\mathrm{n}}$ component again after the second focal point. The effect of anisotropy in this case is to change the effective material parameter abruptly in the two focal points.

We can model the effect of the discontinuity of the material on the internal electric field by imagining a small path segment on the $x$-axis so that the path crosses one of the focal points from the extra-focal region to the focal range. Because $\mathbf{u}_{x}$ is the path's tangent vector, the quantities $\mathbf{u}_{x} \cdot \mathbf{D}$ and $\mathbf{u}_{x} \times \mathbf{E}$ need to be continuous along the path. In particular, the continuity of the $x$-component of the electric flux density implies $\epsilon_{\mathrm{n}} E_{\text {point }}=\epsilon_{\mathrm{t}} E_{\text {foc }}$, where the $x$-component of the field, of course, represents the totality of the $x$-directed field.

On the other hand, when the field is perpendicular and pointed to the direction of $\mathbf{u}_{y}$ the situation is different. The continuity of the $x$-component of the electric flux density still applies, but is irrelevant because it turns out that the field is oriented towards the positive $y$-direction throughout the focal range. The orientation of the field follows from the mirror symmetry of the inclusion with respect to the $y$-axis and from the fact that the field is constant in the focal range. The continuity conditions on the path segment now imply that the $y$-component of the electric field has to be continuous in the major axis. Because the $y$-component represents the whole of the existing field in the focal range, it follows that the field in the focal range coincides with the field in the immediate vicinity of the focal range: $\mathbf{E}_{\mathrm{point}, \perp}=\mathbf{E}_{\mathrm{foc}, \perp}$.

\section{EFFECTS OF RADIAL ANISOTROPY}

The polarizability formulas (14) and (16), that we developed in Sec. III B, allow us to study the interplay of material and geometry in creating the electric response of the inclusion. In particular, we can study the effect of material and geometry on the anisotropy of the inclusion's polarizability. This anisotropy of polarizability is manifest in the polarizability dyadic $\overline{\bar{\alpha}}$ where a disparity between the components $\alpha_{\|}$and $\alpha_{\perp}$ indicates that the polarizability depends on the orientation of the excitation field $\mathbf{E}^{\mathrm{p}}$.

We find that the joint effect of material and geometry on the anisotropy of polarizability can be two-fold. First, the anisotropy in material can enhance the anisotropy of polarizability when the geometry is near-symmetric. The anisotropy enhancing effect is shown in Fig. 3. Second, the anisotropy in material can attenuate the anisotropy in polarizability when the geometry markedly deviates from rotation symmetry. The anisotropy attenuating effect is show in Fig. 4. Both

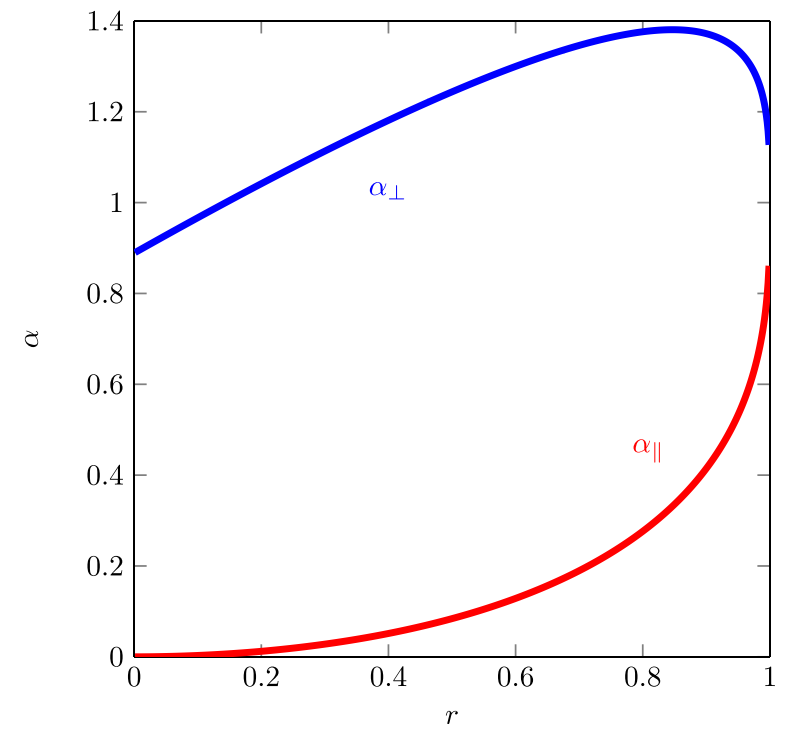

FIG. 3. Enhanced anisotropy of polarizability. The permittivities are $\epsilon_{\mathrm{n}}=9$ and $\epsilon_{\mathrm{t}}=1$.

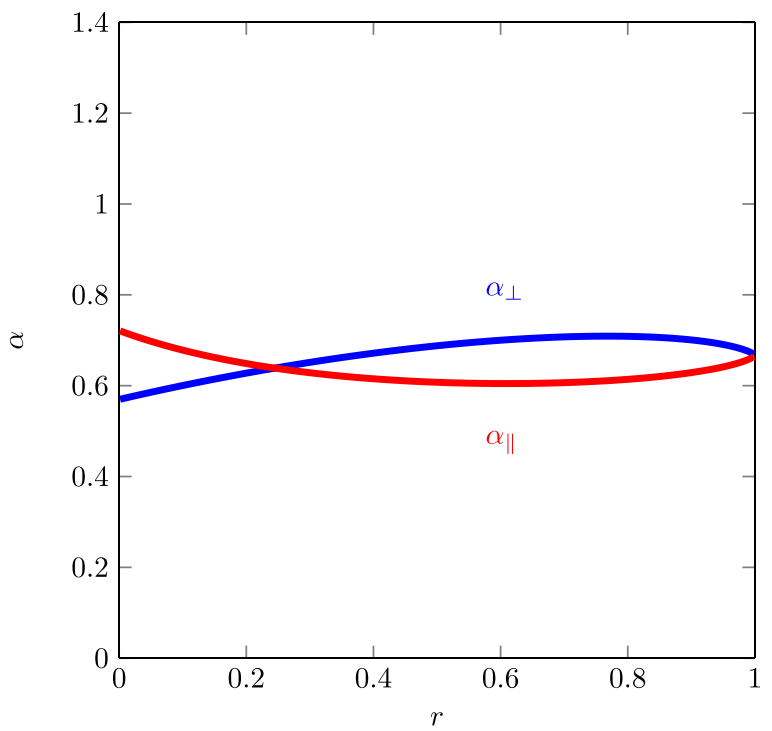

FIG. 4. Attenuated anisotropy of polarizability. The permittivities are $\epsilon_{\mathrm{n}}=$ $2 \sqrt{1.35}$ and $\epsilon_{\mathrm{t}}=2 / \sqrt{1.35}$.

in Figs. 3 and 4, the material parameters are held constant while the geometry varies from needle-like, $r=0$, to circular, $r=1$.

In Fig. 3, an anisotropic polarizability can be observed when $r=0$. At this value of axis ratio, the polarizability is given by (19) and (20). The anisotropy is expected because the geometry of the inclusion imposes it. However, the anisotropy of polarizability persists even when the geometry of the inclusion becomes nearly symmetric. Even at near-unity values of axis ratio, like $r=0.9$, the inclusion still exhibits a marked anisotropy. The reason for the strong anisotropy enhancement in Fig. 3 is that, even though the inclusion is near-symmetric at its outer layers, the inclusion concentrates energy at its center where the asymmetry of material is most pronounced.

Apart from enhanced anisotropy, elliptic inclusions can also exhibit an opposite effect where the geometry of the inclusion is markedly asymmetric but the polarizability is 
isotropic regardless. In Fig. 4, the inclusion has two identical polarizability components $\alpha_{\|}=\alpha_{\perp}=0.64$ when $r=0.245$. But when the anisotropy vanishes for a highly asymmetric inclusion and we know that the anisotropy must vanish regardless of $\epsilon_{\mathrm{n}}$ and $\epsilon_{\mathrm{t}}$ when the inclusion is circular, $r=1$, it is viable to expect that the anisotropy of the inclusion is relatively low at the intermediate values of the axis ratio $r$. Indeed, Fig. 4 manifests only $26 \%$ maximum relative difference between the polarizability components $\alpha_{\|}, \alpha_{\perp}$.

\section{CLOAKS AND CONCENTRATORS}

Existing research on radially anisotropic inclusions has shown that many canonical inclusions can serve as cloaks when the material parameters are adjusted suitably. $8,9,21,22$ They are also known to exhibit field concentration. One of these field concentrating and cloaking canonical inclusions is the radially anisotropic circle, ${ }^{8}$ which is a special case of the subject matter of the present study. In particular, a combination of material parameters $\epsilon_{\mathrm{t}}<\epsilon_{\mathrm{n}}$ produces inclusions that have been referred to as porcupic and that are known to exhibit concentration. The contrasting combination $\epsilon_{\mathrm{n}}<\epsilon_{\mathrm{t}}$ produces inclusions that have been referred to as bulbic and that are known to enable cloaking. ${ }^{21}$ This suggests a potential cloaking and concentration properties also for the radially anisotropic ellipse. The bulbic ellipse does, in fact, turn out to facilitate cloaking. In addition, the porcupic ellipse can form a field concentrator for a suitable excitation field.

In Sec. IV, we concluded that the polarizability of a radially anisotropic needle coincides with that of a homogeneous needle, according to (21), so that the homogeneous permittivity depends on the orientation of the excitation field. We also concluded that when the inclusion becomes circular, the polarizability in (22) coincides with that of a homogeneous circle with the homogeneous permittivity $\left(\epsilon_{\mathrm{n}} \epsilon_{\mathrm{t}}\right)^{1 / 2}$. If we substitute the values $\epsilon_{\mathrm{n}}=0.1$ and $\epsilon_{\mathrm{t}}=2.5$, we note that the parallel polarizability $\alpha_{\|}$changes from negative to positive when the geometry changes from needle to circle. Similarly, if we choose $\epsilon_{\mathrm{n}}=0.1$ and $\epsilon_{\mathrm{t}}=40$, the perpendicular polarizability changes from negative to positive upon the same transformation of geometry. In both cases, the continuity of the polarizability curve implies that the polarizability has to pass through zero at some value of axis ratio $0<r<1$ which, with the above choices of permittivity components, is $r=0.5$ in both cases.

The vanishing polarizability $\alpha_{\|}$at the intersection of the curve with $x$-axis implies that the object becomes invisible to an observer of static fields when the excitation is uniform. Because the coefficients of the perturbation field in (14) and (16) depend linearly on the polarizability $\overline{\bar{\alpha}}$, we conclude that a vanishing polarizability implies a vanishing perturbation field. Thus if the perturbation vanishes in the far-region of the inclusion, it must also vanish in the proximity of the inclusion.

In addition to invisibility, the former research ${ }^{21}$ leads us to expect that the elliptic inclusion exhibits cloaking in the sense outlined by Pendry, ${ }^{23}$ namely, that it can conceal an object so that an external observer only sees vacant space where the concealed object is. When we take the parallel excitation field, the perturbation field vanishes with the combination of parameters $\epsilon_{\mathrm{t}}=0.1, \epsilon_{\mathrm{n}}=2.5, r=0.5$. The potential distribution in Fig. 5 shows that an inclusion with these specifications efficiently repulses static electric field from its focal range. We could therefore surreptitiously house a suitably small object in the field-depleted "sweet spot" of the cloak, and the object would successfully elude an external, statics-bound observer.

Similarly, we can erect a cloaking structure to hide an object from a perpendicular excitation field. The asymmetry of the inclusion implies that the material parameters be different from the above parallel case. We achieve the cloaking effect with the combination of parameters $\epsilon_{\mathrm{n}}=0.1, \epsilon_{\mathrm{t}}=40$, and $r=0.5$. We find that the sweet spot expands from the one found for the parallel field. A possible explanation for

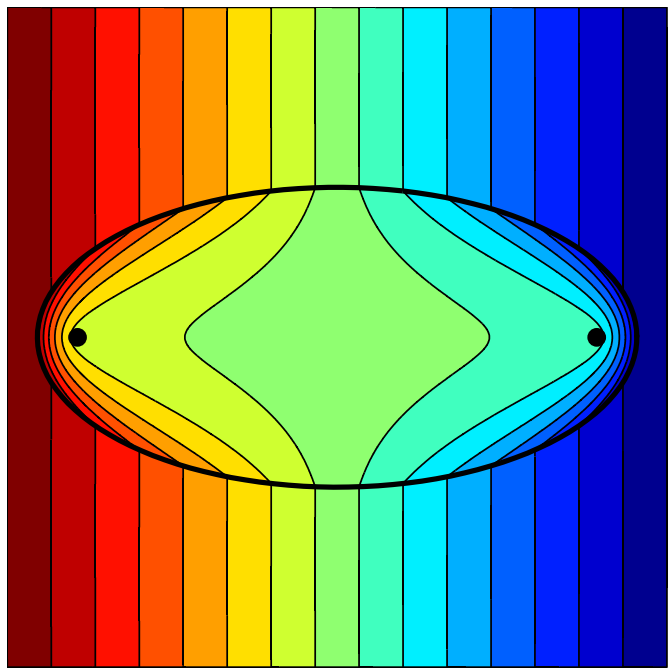

(a)

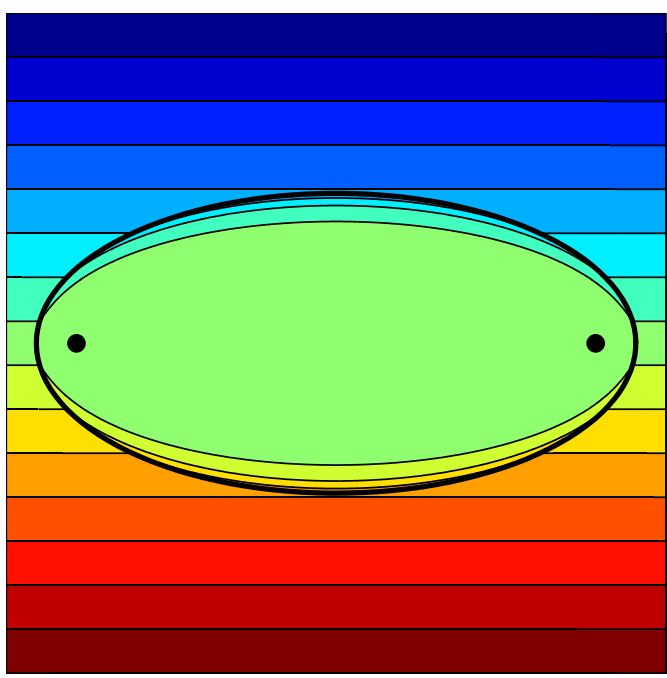

(b)

FIG. 5. The cloaking effect of an RA ellipse when impinged by a uniform and static excitation field. The potential distribution is illustrated for two cases. The top panel shows an ellipse in a parallel excitation field. This ellipse has the axis ratio $r=0.5$ and the permittivities $\epsilon_{\mathrm{n}}=0.1$ and $\epsilon_{\mathrm{t}}=2.5$. The choice of material parameters causes the perturbation field to vanish. The bottom panel illustrates an ellipse in a perpendicular excitation field. The axis ratio is again $r=0.5$, but the permittivities are $\epsilon_{\mathrm{n}}=0.1$ and $\epsilon_{\mathrm{t}}=40$. Again the perturbation vanishes. 
the expansion of the sweet spot is that in the perpendicular case the excitation potential changes less within the inclusion than in the parallel case. Another plausible explanation is that a higher permittivity ratio $\epsilon_{\mathrm{t}} / \epsilon_{\mathrm{n}}$ causes a stronger cloaking effect.

Apart from a qualitative inspection of the size of the sweet spot, there is another more objective way to assess the strength of the cloaking effect. We can calculate the strength of the electric field in the focal range using (23) and (25) and compare the result to the magnitude of the excitation field to see how efficiently the inclusion repels fields from its center. It turns out that, from the two cloaking configurations that we considered, the one with the larger sweet spot also has weaker

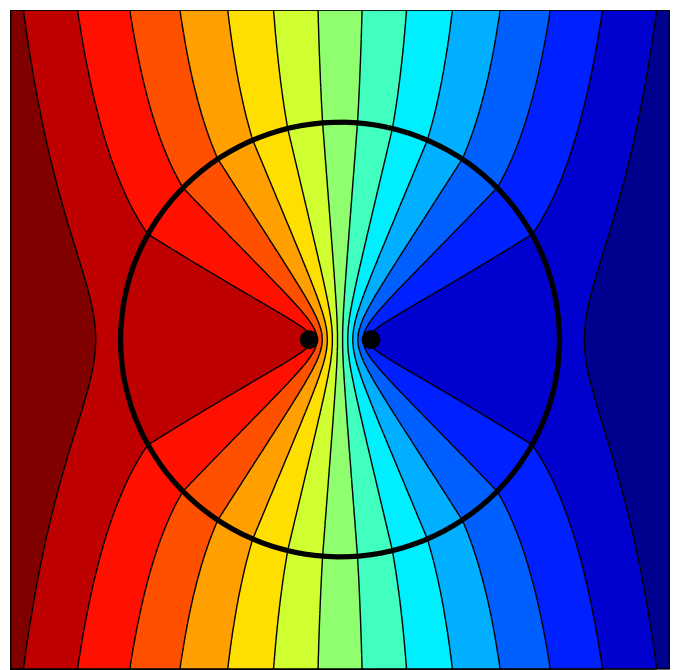

(a)

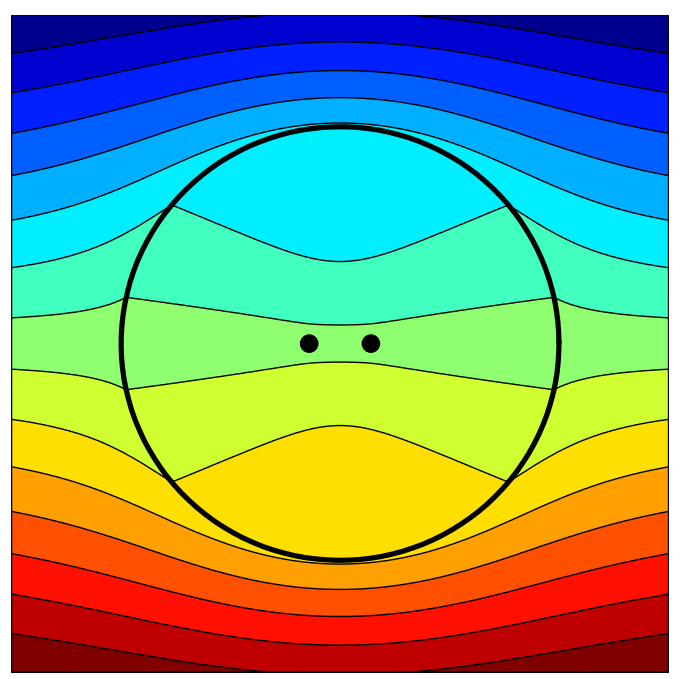

(b)

FIG. 6. The field concentration effect of an RA ellipse when impinged by a uniform and static excitation field. The illustrated potential distributions indicate a drastic difference between the inclusion's response to a parallel field (on the top) as opposed to the response to a perpendicular field (on the bottom). In both cases the axis ratio is $r=0.99$ and the permittivities are $\epsilon_{\mathrm{n}}=10$ and $\epsilon_{\mathrm{t}}=0.1$. Still, the concentration of electrostatic energy is much intenser when the field is parallel as opposed to perpendicular. Furthermore, the polarizability preserves its magnitude but changes from $\alpha_{\|}=-1.17$ to $\alpha_{\perp}=1.17$. The response of the inclusion is in this respect highly anisotropic, especially considering its nearly circular perimeter. electric field in the focal range, because the respective field strengths are $\mathbf{E}_{\mathrm{foc}, \|}=0.14 \cdot \mathbf{E}^{\mathrm{p}}$ and $\mathbf{E}_{\mathrm{foc}, \perp}=0.00039 \cdot \mathbf{E}^{\mathrm{p}}$.

When it comes to field concentrators, the anisotropy of the scatterer is even more manifest. The inclusion in Fig. 6 exhibits a concentration effect, which is strikingly dependent on the orientation of the excitation field, even when the material parameters $\epsilon_{\mathrm{n}}=10$ and $\epsilon_{\mathrm{t}}=0.1$ are the same in top and bottom panels and the perimeter of the inclusion deviates from circular symmetry remarkably little, with the elliptic axis ratio $r=0.99$.

A likely resolution to this conundrum lies in Equations (23)-(25). When the field is parallel, the discontinuity of the electric field at the focal points drives more of the equipotential lines to the focal range and thereby causes concentration of electrostatic energy. In the perpendicular case, the field is continuous at the focal points, so that the relative mildness of concentration effect in the extra-focal region comes over to the focal range.

Even though it looks as if the result in Fig. 6 would pose obstacles for creating concentrators out of porcupic circles, the real prospects are less adverse. Regarding the circular concentrators, the present study proposes a very special kind of perturbation where most of the perturbation takes place near the center of the inclusion. Perturbations in the center are expected to diminish the concentration effect because the high energy density makes that region particularly critical. Random perturbations to a circular porcupic concentrator would be unlikely to diminish field concentration as drastically as the transformation into an elliptic geometry.

Another property of the concentrator in Fig. 6 is that its perpendicular polarizability $\alpha_{\perp}$ is exactly the opposite of the parallel polarizability $\alpha_{\|}$. We can explain the property in qualitative terms when we consider the polarizability of an RA circle (22). The polarizability of the circle vanishes when $\epsilon_{\mathrm{n}} \epsilon_{\mathrm{t}}=1$, which suggests that, even when the inclusion deviates from symmetry, its polarizability should vanish on an average over all polarizations of the excitation field $\mathbf{E}^{\mathrm{p}}$. However, a more exact explanation follows when we substitute $\epsilon_{\mathrm{n}} \epsilon_{\mathrm{t}}=1$ to (14) and (16). Then, we find that $\alpha_{\|}$and $\alpha_{\perp}$ are opposites, not only for near-unity values of $r$, but for the whole range of permissible axis ratios.

\section{CONCLUSION}

In this article, we examined the scattering properties of an elliptic, radially anisotropic inclusion in a static, uniform excitation field. We found that the problem could be divided into two parts, one concerning an excitation field parallel to the focal range of the inclusion and another concerning a perpendicular excitation field. The treatment of both parts of the problem, in Secs. III B and III C, followed similar courses with minor distinctions. These distinctions originated from a disparity between the two angular dependencies of the excitation fields involved. After we obtained the solution for the two cases, a general solution for an arbitrary uniform excitation field could then be represented as a linear combination of two known solutions.

The results were validated by comparing them with existing results for special cases. The first mode of validation 
was to compare the ellipse formulas with those for a radially anisotropic circle. In this case, we found a perfect match with the results. The second mode of validation was to elongate the ellipse into a needle. Our hypothesis was that the inclusion would become effectively homogeneous upon elongation. A comparison with a homogeneous needle confirmed the hypothesis.

When we studied the way that the polarizability of an ellipse depends on the two material parameters, we found that combinations of parameters exist that make the inclusion lose its perturbation field. This and existing research ${ }^{8,9,21,22}$ on similar inclusions made us expect a potential cloaking property of the ellipse. When we investigated the matter, we found that it is indeed possible to have the inclusion's perturbation field vanish, so that the internal field near the focal range vanishes concurrently. We found that the cloaking effect depends on the orientation of the excitation field, so the cloak is more efficient when the excitation field is perpendicular.

Aside cloaks, we also studied their field enforcing counterpart, the field concentrators. We found that the concentrators, like the cloaks, were anisotropic in their response to the orientation of the excitation field. Furthermore, we found that the anisotropy even affected seemingly symmetric geometries, like an ellipse with the axis ratio $r=0.99$. In particular, the concentrator seemed to condense electrostatic energy when the field was parallel, but it failed to achieve a substantial energy concentration when the perpendicular fields were involved. We attempted to offer some discussion about the viable reasons for the perhaps unexpected disparity.

The study of radially anisotropic ellipses takes us one step further in the pursuit novel results in engineering and mathematics. Besides establishing a new touchstone in the self-correcting network of test cases in electrostatics, it also suggests approaches to intriguing engineering challenges, like cloaking and field concentration. The research stands of well-established axioms of electromagnetic field theory, and can thus be regarded as fairly solid grounds for validating numerical methods and assessing the accuracy of approximate methods. The continuing relevance of closed form solutions will ascertain that the analytic study of canonical shapes will continue to pursue its unrelenting and informative course.

${ }^{1}$ C. Yeh, "Elliptical dielectric waveguides," J. Appl. Phys. 33, 3235-3243 (1962).
${ }^{2}$ P. Moon and D. E. Spencer, Field Theory Handbook, 2nd ed. (SpringerVerlag, New York, USA, 1988).

${ }^{3}$ R. Pérez-Enriquez, I. Marín-Enriquez, J. L. Marín, and R. Riera, "Chebyshev functions and their use for two-dimensional electrostatic problems in elliptic coordinates," WSEAS Trans. Math. 4, 56 (2005).

${ }^{4}$ J. Marín, I. Marín-Enriquez, R. Riera, and R. Pérez-Enriquez, "On the potential of an infinite dielectric cylinder and a line of charge: Green's function in an elliptic coordinate approach," Rev. Mex. Fis. 53, 41-47 (2007).

${ }^{5}$ H. Ragheb, L. Shafai, and M. Hamid, "Plane wave scattering by a conducting elliptic cylinder coated by a nonconfocal dielectric," IEEE Trans. Antennas Propag. 39, 218-223 (1991).

${ }^{6}$ J. H. Richmond, "Scattering by a conducting elliptic cylinder with dielectric coating," Radio Sci. 23, 1061-1066, doi:10.1029/RS023i006p01061 (1988).

${ }^{7}$ F. Mangini, N. Tedeschi, F. Frezza, and A. Sihvola, "Homogenization of a multilayer sphere as a radial uniaxial sphere: features and limits," J. Electromagn. Waves Appl. 28, 916-931 (2014).

${ }^{8} \mathrm{H}$. Kettunen, H. Wallén, and A. Sihvola, "Cloaking and magnifying using radial anisotropy," J. Appl. Phys. 114, 044110 (2013).

${ }^{9}$ Y. Ni, L. Gao, and C.-W. Qiu, "Achieving invisibility of homogeneous cylindrically anisotropic cylinders," Plasmonics 5, 251-258 (2010).

${ }^{10}$ J. Roth and M. Dignam, "Scattering and extinction cross sections for a spherical particle coated with an oriented molecular layer," J. Opt. Soc. Am. 63, 308-311 (1973).

${ }^{11}$ J. C. de Munck, "The potential distribution in a layered anisotropic spheroidal volume conductor," J. Appl. Phys. 64, 464-470 (1988).

${ }^{12} \mathrm{~K}$. Wong and H. Chen, "Electromagnetic scattering by a uniaxially anisotropic sphere,” IEE Proc. H 139, 314-318 (1992).

${ }^{13}$ J.-E. Sten, "DC fields and analytical image solutions for a radially anisotropic spherical conductor," IEEE Trans. Dielectr. Electr. Insul. 2, 360-367 (1995).

${ }^{14}$ Y. Ni, D. Gao, Z. Sang, L. Gao, and C.-W. Qiu, "Influence of spherical anisotropy on the optical properties of plasmon resonant metallic nanoparticles," Appl. Phys. A 102, 673-679 (2011).

${ }^{15}$ Y. Ni, L. Gao, A. Miroshnichenko, and C.-W. Qiu, "Non-Rayleigh scattering behavior for anisotropic Rayleigh particles," Opt. Lett. 37, 3390-3392 (2012).

${ }^{16}$ T. Rimpiläinen, H. Wallén, H. Kettunen, and A. Sihvola, "Electrical response of systropic sphere," IEEE Trans. Antennas Propag. 60, 5348-5355 (2012).

${ }^{17}$ T. Rimpiläinen, M. Pitkonen, H. Wallén, H. Kettunen, and A. Sihvola, "General systropy in spherical scatterers," IEEE Trans. Antennas Propag. 62, 327-333 (2014).

${ }^{18}$ See supplementary material at http://dx.doi.org/10.1063/1.4939158 for numerical validation.

${ }^{19}$ A. Sihvola, Electromagnetic Mixing Formulas and Applications (The Institution of Electrical Engineers, London, England, 1999), p. 284.

${ }^{20}$ T. Rimpiläinen, "Electric response of an anisotropic sphere," Master's thesis (Aalto University, School of Electrical Engineering, 2011).

${ }^{21} \mathrm{~T}$. Rimpiläinen, H. Wallén, and A. Sihvola, "Radial anisotropy in spheroidal scatterers," IEEE Trans. Antennas Propag. 63, 3127-3135 (2015).

${ }^{22} \mathrm{H}$. Wallén, H. Kettunen, and A. Sihvola, "Anomalous absorption, plasmonic resonances, and invisibility of radially anisotropic spheres," Radio Sci. 50, 18-28, doi:10.1002/2014RS005534 (2015).

${ }^{23}$ J. Pendry, D. Schurig, and D. Smith, "Controlling electromagnetic fields," Science 312, 1780-1782 (2006). 